\title{
INFLUENCE OF CHANNEL WIDTH VARIATIONS ON STRUCTURE AND MIXING OF INTERNAL HYDRAULIC JUMPS
}

\author{
Michael Ganotaki ${ }^{1}$, Kelly Anne Ogden ${ }^{1 *}$ \\ ${ }^{1}$ Department of Mechanical and Materials Engineering, Western University, London, Canada \\ *kogden3@uwo.ca
}

\begin{abstract}
The effect of channel width variations on the structure and mixing of internal hydraulic jumps is investigated by simulating internal hydraulic jumps over a sill that is coincident with a contraction. Density variance production across the jump is used to quantify the mixing. Simulations indicate that most jumps experience lateral flow separation and the main effect of channel width variations on the jump is to increase the velocity of flow in the jump region.
\end{abstract}

Keywords-hydraulic jump; waves; mixing; channel width variation

\section{INTRODUCTION}

Internal hydraulic jumps, which occur within a stratified fluid body, are more complex than surface hydraulic jumps due to the associated change in surface height, or pressure, across the jump, and due to the mixing across density surfaces within the jump. Internal hydraulic jumps occur in many flows in the environment, from coastal channels, to the deep ocean, to atmospheric flow over mountains [1]-[3]. They are important due to the energy dissipation and mixing that they cause. For example, the internal hydraulic jump in Knight Inlet dissipates one third of the barotropic tidal energy in the channel [3]. Internal hydraulic jumps in coastal waters are often approximated as two-layered and two-dimensional to simplify the analysis of the flow [5]-[7]. Conversely, very detailed analyses of a few specific locations, such as Knight Inlet, have been conducted [4]. However, the general influence of important flow parameters, such as the channel width variations, have yet to be categorized. To address this gap in knowledge, this paper examines the effect of cross-channel topography on the mixing and qualitative structure of internal hydraulic jumps.

\section{Simulation SetuP}

A set of simualtions were conducted to assess the influence of channel width variation on jump structure and mixing. The topographies include coincident sills and contractions. The channel width at the sill and the rate at which the channel narrows were both varied. The scaled density field, $T=\frac{\rho-\rho_{\min }}{\rho_{\max }-\rho_{\min }}$, at the initial time is shown for an example

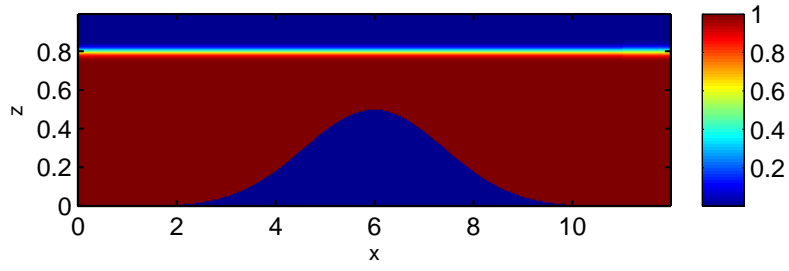

Fig. 1. Scaled density field, $T$, at the initial time, $t=0$ for case 1 , with topography parameters $D=4$ and $W_{s}=1.5$.

simulation in figure I, which also shows the topography along the centreline of the channel in blue. The simulations are non-dimensionalized using the total depth $H$ as the characteristic length scale and $\sqrt{g^{\prime} H}=\sqrt{\frac{\Delta \rho}{\rho_{0}} g H}$ as the characteristic velocity scale.

Simulations were conducted using Gerris [8], which solves the incompressible Boussinesq Navier-Stokes equations:

$$
\begin{aligned}
\frac{\partial \vec{u}}{\partial t}+\vec{u} \cdot \nabla \vec{u} & =-\frac{1}{\rho_{0}} \nabla p^{\prime}+\nu \nabla^{2} \vec{u}-g^{\prime} \hat{k} \\
\frac{\partial \rho^{\prime}}{\partial t}+\vec{u} \cdot \nabla \rho^{\prime} & =\kappa \nabla^{2} \rho^{\prime} \\
\nabla \cdot \vec{u} & =0 .
\end{aligned}
$$

The velocity $\vec{u}=(u, v, w)$, has three components: $u$ is along channel, $v$ is transverse, and $w$ is vertical. The density deviation is $\rho^{\prime}, p^{\prime}$ is the pressure deviation from hydrostatic, $\nu$ is the kinematic viscosity, $\kappa$ is the density diffusivity, $g^{\prime}=\frac{\rho-\rho_{0}}{\rho_{0}} g$ is the reduced gravity, and $\hat{k}$ is the unit vector in the vertical direction.

Simulations employ a viscosity,

$$
\nu=1.8 \cdot 10^{-6} \exp -\frac{\left(z-h_{T}\right)^{2}}{0.01}
$$

which decays exponentially in the vertical, away from topography. This allows a no-slip boundary condition to be implemented along the channel walls, making flow separation from the channel walls possible, without introducing 
significant viscosity in the flow interior.

The initial flow is stationary and stably stratified, with a thin hyperbolic tangent density transition between two largely homogeneous layers of fluid, described by

$$
T(t=0)=\frac{1}{2}(1-\tanh (\lambda(z-0.8))) .
$$

The velocity at the inlet ramps up from zero in time, reaching and maintaining the steady forcing value after a nondimensional time of $t_{T}$ :

$$
U(x=0)= \begin{cases}U_{0} \sin \frac{2 \pi t}{t_{T}} & , t<\frac{t_{T}}{4} \\ U_{0} & , t>\frac{t_{T}}{4}\end{cases}
$$

where $t_{T}=5$ and $U_{0}=0.1$ in all simulations discussed here. The outlet condition is $\frac{\partial(u, v, w)}{\partial x}=0$, and the pressure is set to the hydrostatic pressure.

The topography is described by

$$
h_{B}=h_{T} e^{\frac{-\left(\left(x_{0}-x\right)^{2}\right.}{D}}+W_{s} \frac{\left(y-y_{0}\right)^{2}}{W^{2}} e^{-\frac{\left(x_{0}-x\right)^{2}}{D}},
$$

where $h_{T}=0.5, x_{0}=6, y_{0}=0.5$, and $W=0.5$. $W_{s}$ and $D$ vary between simulations, controlling the width of the channel at the sill and the rate of narrowing, respectively. We expect the rate of narrowing to be the most important parameter in understanding the effect of channel width variations on the jump. A narrower sill will result in a faster flow, which affects mixing in the jump regardless of channel width variations. Therefore, three simulations, all with $W_{s}=1.5$, and with $D=1,2$, and 4 are the main focus of this work.

Simulations employ an isotropic Cartesian grid with 128 grid points in the vertical and cross-channel directions, which previous work has shown to provide sufficient resolution for this type of flow [4], [7].

\section{RESULTS}

A first mode internal hydraulic jump forms downstream of the sill in all cases, as shown in figures II and III, which show a density isosurface colored by the along channel velocity, and the scaled density field on a horizontal and a vertical slice through the flow, for two cases with different channel width narrowing rates $(D=4$ and $D=1)$.

All simulation results show laterally separating flow from the sides of the channel, suggested by the vorticity and streamlines shown in figure IV. The streamlines along the side of the channel from about $x=7.5$ to $x=8$ show closed regions of recirculation. The vertical vorticity of the flow in these areas is consistent with recirculation in that area, although the vorticity field is patchy because the average was calculated from only about 15 instantaneous flow results for the horizontal section data.
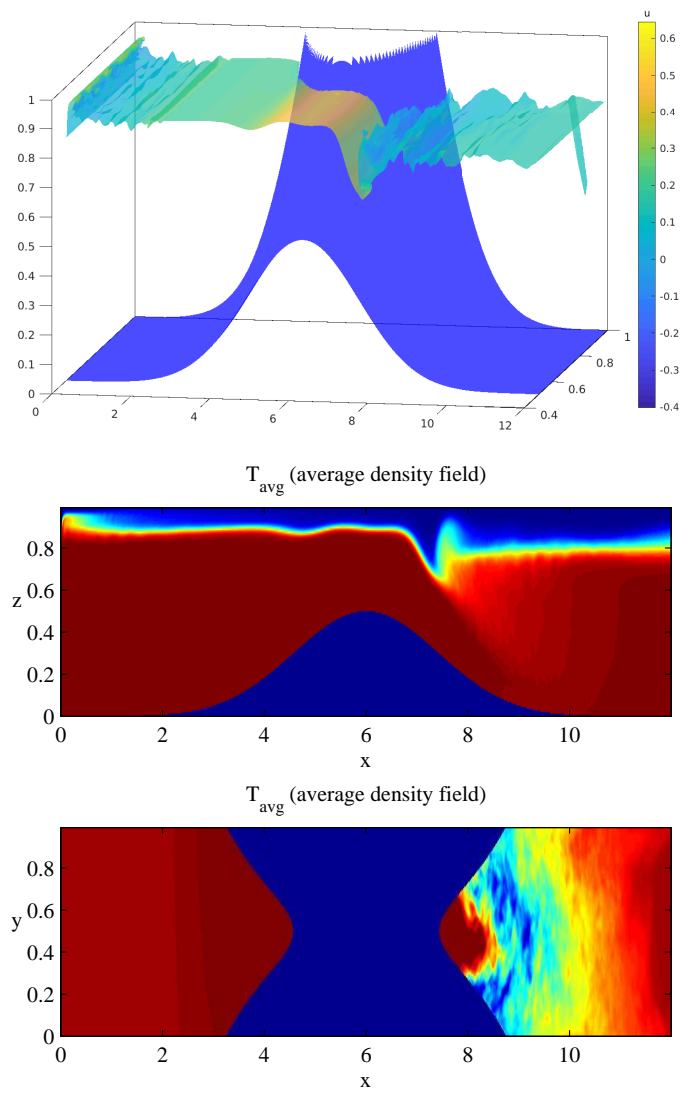

Fig. 2. Top: 3-D isosurface of $T=0.5$ colored by $u$ shown with topography for half of the cannel. Middle: vertical along channel slice at $y=0.5$ showing $T$ field. Bottom: horizontal slice at $z=0.3$ showing $T$ field. Case 1 has $W_{s}=1.5$ and $D=4$.

Mixing is quantified by calculating the integral of the scalar variance production in the region of the jump. The scalar variance is governed by [11]:

$\frac{1}{2} \frac{\partial}{\partial t} \overline{\theta^{2}}+\frac{U_{i}}{2} \frac{\partial}{\partial x_{j}} \overline{\theta^{2}}+\overline{u_{j} \theta} \frac{\partial \Theta}{\partial x_{j}}+\frac{1}{2} \frac{\partial}{\partial x_{i}} \overline{u_{i} \theta^{2}}+D \overline{\frac{\partial \theta}{\partial x_{i}} \frac{\partial \theta}{\partial x_{i}}}=0$.

The cumulative area integral of the scalar variance production across the jump, $\overline{u_{j} T} \frac{\partial \bar{T}}{\partial x_{j}}$, where scaled density $T$ is the scalar of interest, quantifies the amount of mixing that occurs within the jump itself [9]. Note that larger magnitudes indicate larger mixing, while an increase in scalar variance produces a negative value of $\int_{a}^{b} \int_{0}^{H} \frac{u_{j} T}{\partial x_{j}} d z d x$. Applying the integral across the jump allows us to assign a single value of mixing to each jump. The amount of mixing for several jumps is compared to the channel width at the contraction $\left(W_{s}\right)$ and the narrowing $(D)$ in figure V. Scalar variance production in the along-channel and vertical directions, $\overline{u T} \frac{\partial \bar{T}}{\partial x}+\overline{w T} \frac{\partial \bar{T}}{\partial z}$ is calculated separately from scalar variance production in the along- and cross-channel directions, $\overline{u T} \frac{\partial \bar{T}}{\partial x}+\overline{v T} \frac{\partial \bar{T}}{\partial y}$. This was done because data must be saved frequently to calculate reliable statistics, and saving data over the entire computational domain with this frequency is difficult. Approx- 


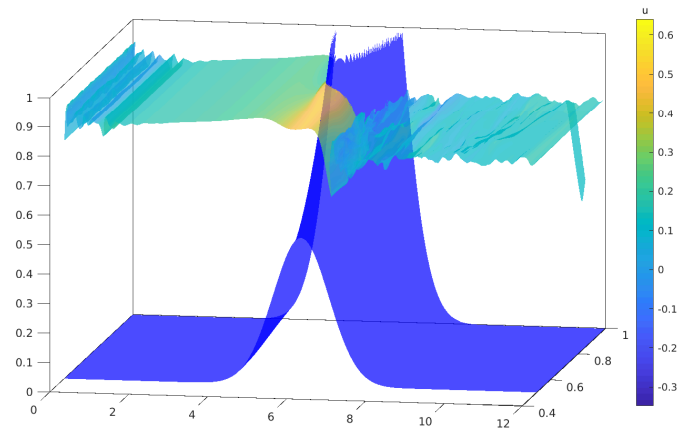

$\mathrm{T}_{\text {avg }}$ (average density field)

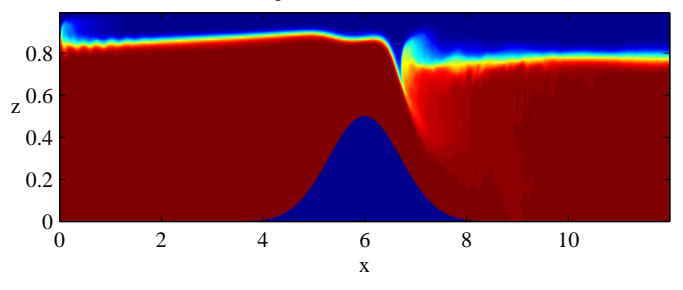

$\mathrm{T}_{\text {avg }}$ (average density field)

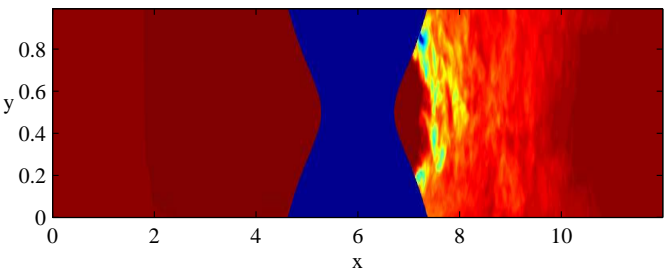

Fig. 3. Top: 3-D isosurface of $T=0.5$ colored by $u$ shown with topography for half of the cannel. Middle: vertical along channel slice at $y=0.5$ showing $T$ field. Bottom: horizontal slice at $z=0.3$ showing $T$ field. Case 2 has $W_{s}=1.5$ and $D=1$.

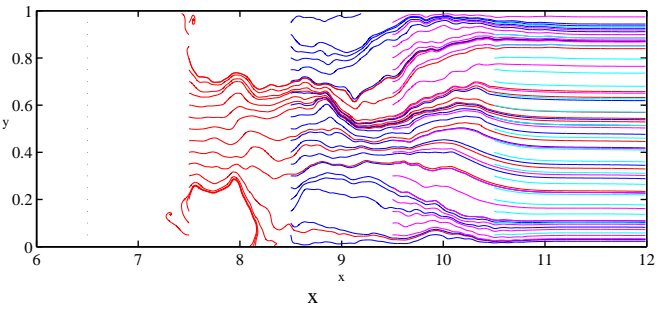

$\omega$

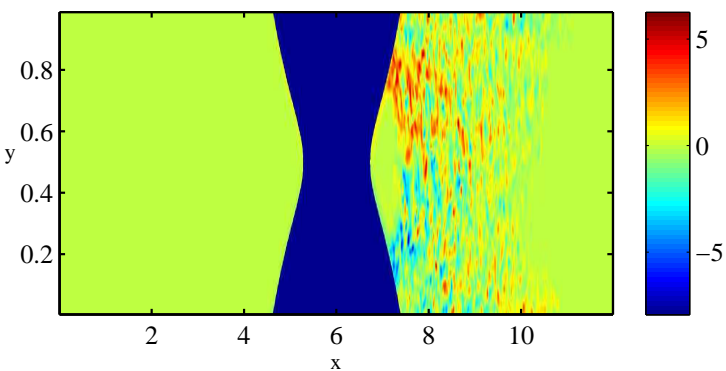

Fig. 4. Top: streamlines in the $x-y$ plane at $z=0.3$ for case $2\left(W_{s}=1.5\right.$ and $D=1$ ). Bottom: Vertical component of vorticity, $\omega$.

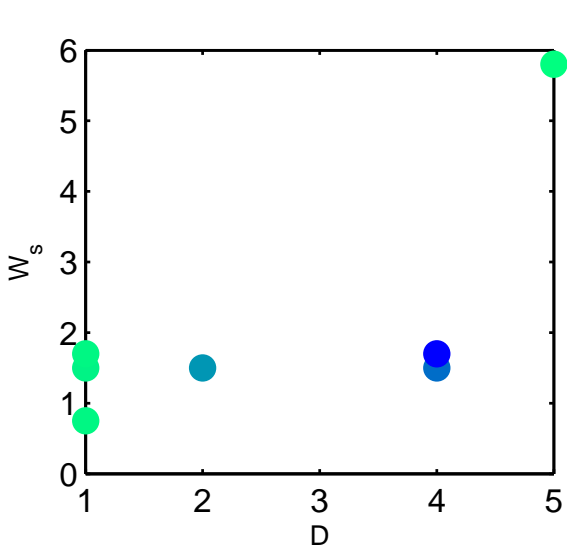

$\times 10^{-3}$
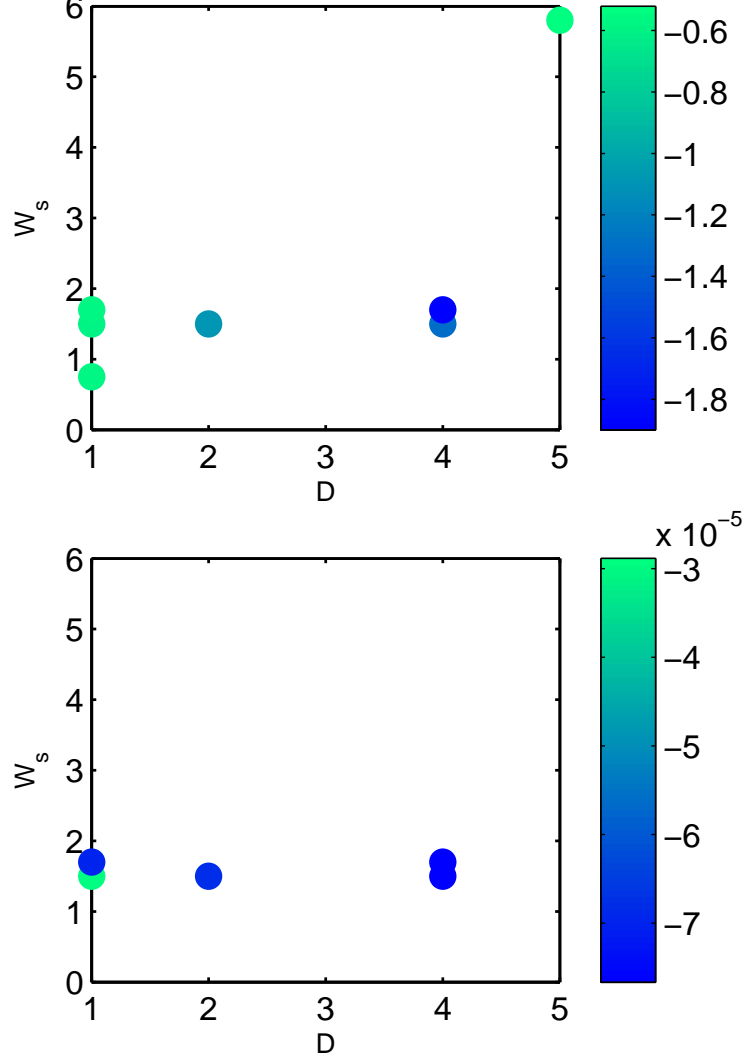

Fig. 5. Scalar variance production components in the vertical and along channel direction $\left(\overline{u T} \frac{\partial \bar{T}}{\partial x}+\overline{w T} \frac{\partial \bar{T}}{\partial z}\right.$, top) and scalar variance production components in the cross-channel and along channel directions ( $\overline{u T} \frac{\partial \bar{T}}{\partial x}+\overline{v T} \frac{\partial \bar{T}}{\partial y}$, bottom), plotted relative the the rate of narrowing. Case 1 from figure III is at $(4,1.5)$ and case 2 is at $(1,1.5)$. Simulations without explicit viscosity (free-slip boundary condition) are offset by $W_{s}+0.2$ so both results are visible.

imately 50 instantaneous results are included in the vertical section averaging. Comparing the two separate calculations shows that mixing primarily occurs in the vertical direction since $\overline{u T} \frac{\partial \bar{T}}{\partial x}+\overline{w T} \frac{\partial \bar{T}}{\partial z}$ is an order of magnitude larger than $\overline{u T} \frac{\partial \bar{T}}{\partial x}+\overline{v T} \frac{\partial \bar{T}}{\partial y}$. Larger mixing in the vertical direction is expected since the internal hydraulic jump is a process driven by a vertical density gradients and velocity shears. The lateral mixing is more significantly influenced by the lateral flow separation.

Figure $\mathrm{V}$ shows that the mixing is larger for channels with a gentler rate of narrowing (larger $D$, lower rate of expansion after the jump). This likely occurs because separation occurs in all of the simulated jumps, so the details of separation are not significantly different between jumps, or the differences are not important. Instead, when the channel expands slowly after the sill, the jump occurs in a narrower region, meaning the velocity, and likely the vertical shear, just ahead of the jump are larger. These larger velocities result in "stronger" jumps, which experiences more mixing. The effect of the channel width variation on the mixing 


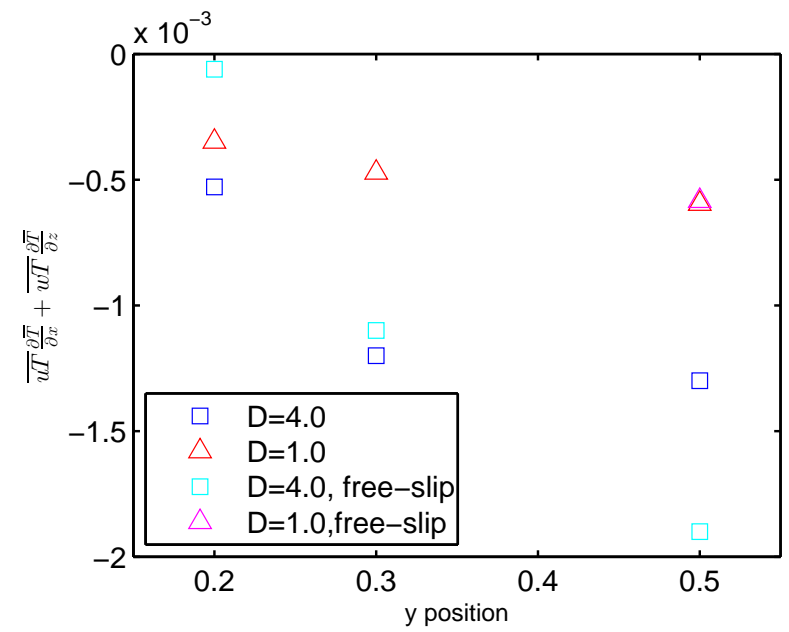

Fig. 6. Scalar variance production components in the vertical and along channel direction calculated at the centreline $(y=0.5)$ and at $y=0.3$ and $y=0.2$. Case $1\left(D=4, W_{s}=1.5\right)$ and case $2\left(D=1, W_{s}=1.5\right)$.

is indirect; the narrower channel creates a stronger jump, resulting in more mixing. The separation does not appear to cause significant direct changes to the mixing and structure of the jump. In channels with lateral channel width variation and separation, the amount of mixing caused by a jump is likely related to the flow speed and vertical shear just ahead of the jump, rather than the lateral flow separation. This hypothesis should be investigated further with additional simulations for comparison.

Understanding how the flow varies across the channel is important in interpreting observational data. Mixing (equal to the cumulative integral of the density variance production) at the vertical midsection of the channel is comparable to, but slightly larger than, mixing at $30 \%$ of the cross-channel distance for cases 1 and 2. The scalar variance production decreases away from the centreline of the channel, as shown in figure VI. Figure VII shows where in the flow density variance is produced. The production from $x=7.5$ to $x=8$ in the vertical section along the centreline of the channel exhibits the largest amount of mixing. The mixing farther from the centre of the channel, and in the horizontal plane, is smaller.

Most of the scalar variance production in the cross-channel direction occurs in the recirculating regions of the jump, as shown in the bottom panel of figure VII. However, the pointwise values, as well as the cumulative integral, is an order of magnitude smaller than values seen in the vertical component.

\section{INVISCID Simulations}

Inviscid simulations equivalent to several of the simulations discussed previously were conducted to determine whether explicit viscosity and a no-slip bottom boundary condition
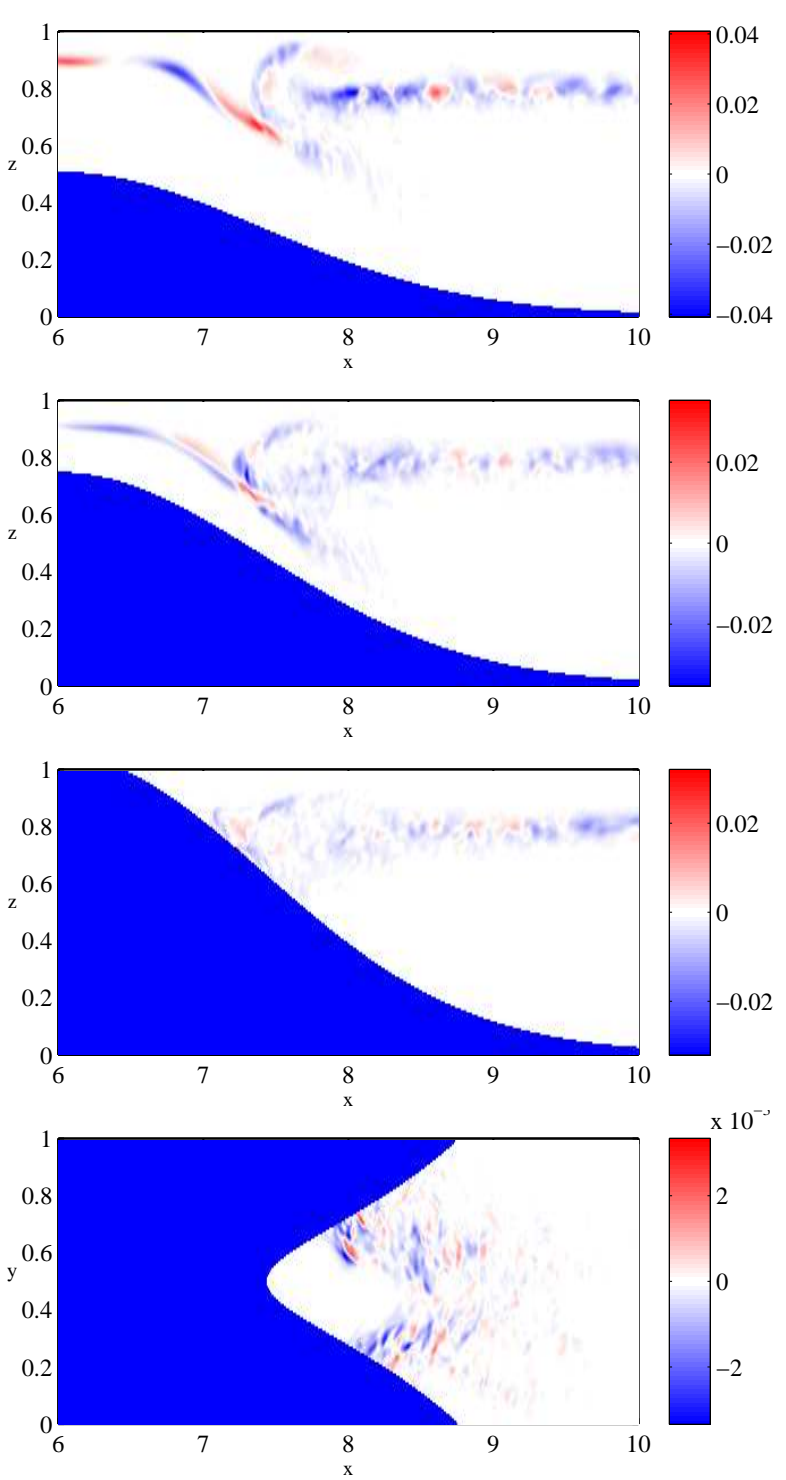

Fig. 7. Scalar variance production components in the vertical and along channel direction calculated at the centreline $(y=0.5)$ and at $y=0.3$ and $y=0.2$, and in a horizontal plane at $z=0.3$. Case $1\left(D=4, W_{s}=1.5\right)$.

is required to generate lateral separation in the channel expansion.

Recirculation regions form despite the free-slip boundary condition, as shown in figure VIII. This contrasts with bottom boundary layer separation, which requires explicit viscosity and a no-slip bottom boundary condition to occur. Lateral separation and recirculation likely occur because the turbulence of the jump is directly adjacent to the channel side walls, allow separation despite the boundary condition. Whereas the turbulence of the jump is vertically located partway between the bottom and upper boundaries of the flow, rather than directly against the bottom boundary. 

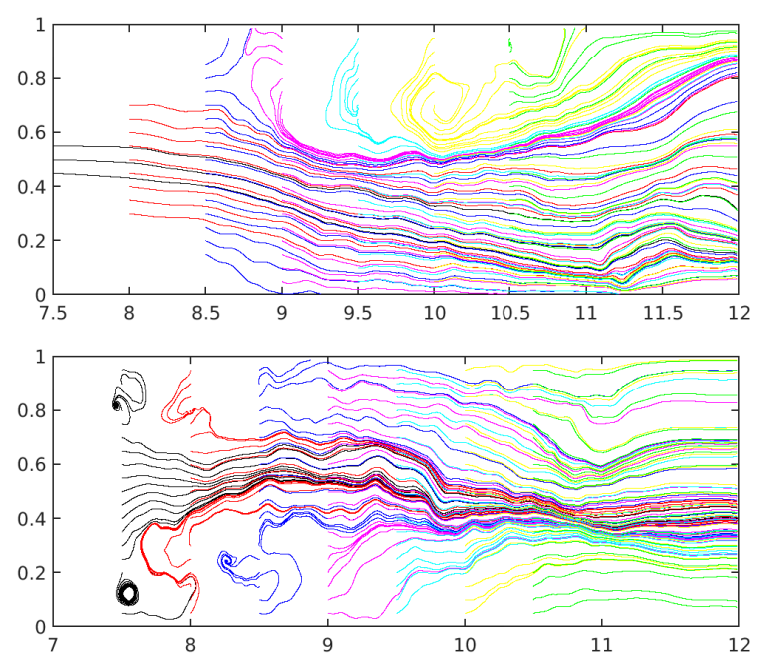

Fig. 8. Streamlines in a horizontal plane at $z=0.3$ for (top) case $1 \mathrm{i}$ ( $D=4$, $W_{s}=1.5$, free-slip boundary condition) and (bottom) case $2 \mathrm{i}(D=1$, $W_{s}=1.5$, free-slip boundary condition) and (bottom).

\section{CONCLUSIONS}

Lateral flow separation occurs in all topographies tested. This is consistent with boundary layer flow theory for flow over a flat plate, which predicts separation at a plate angle of about $18^{\circ}$ [12]; the topographies used all appear to include a topographic region with this angle, resulting in lateral flow separation and recirculation. However, lateral separation also occurs for flows without explicit viscosity, indicating that turbulence from the jump may contribute to the lateral recirculation. This result reinforces the importance of considering channel width variation, which affects the path of the flow through both the topography and the recirculation regions. The flow separation alters the shape of the jump; the channel is effectively narrower for a longer distance downstream of the sill, which forces the jump to occur in a narrower section of the channel, resulting in a higher flow velocity and likely larger upstream shear just ahead of jump. If the upstream shear is larger, mixing is also more significant. This result is consistent with Ogden [10], which showed that upstream shear is the main factor that determines the amount of mixing in the jump. To compare the effect of separation to the effective change in channel shape, a simulation without viscosity and with free-slip conditions along the channel walls, should be conducted.

Although flow recirculation appears to occur in all simulations considered here, the scalar variance production, or mixing, is dominated by the jump rather than by the recirculation regions. This is because density variance increases when fluid of different densities mix together; since the recirculation regions appear to be oriented horizontally, they do not increase the density variance significantly.

This work shows that, when observing a jump in the environment, channel width variations should be considered. Specifically, the variation in mixing across the jump should be considered; the values along the thalweg cannot simply be applied across the entire width of the channel. The variation of density variance production across the jump should be investigated in further detail.

\section{REFERENCES}

[1] D. K. Lilly, "A Severe Downslope Windstorm and Aircraft Turbulence Event Induced by a Mountain Wave," J. Atmos. Science, vol. 35, pp. 59-77, 1978.

[2] K. L. Polzin, K. G. Speer, J. M. Toole, and R. W. Schmitt, "Intense mixing of Antarctic Bottom Water in the equatorial Atlantic Ocean", Nature, vol. 380, pp 54-57, 1996.

[3] J. Klymak and M. Gregg, "Tidally generated turbulence over the Knight Inlet Sill,” J. Phys. Oceanography, vol. 34, pp. 1135-1151, 2004.

[4] K. Lamb, "On boundary-layer separation and internal wave generation at the Knight Inlet sill,” Proc. R. Soc. Lond. A, vol. 460, pp. 2305-2337, 2004.

[5] D.M. Holland, R.R. Rosales, D. Stefanica, and E.G. Tabak, "Internal hydraulic jumps and mixing in two-layer flows," J. Fluid Mech., vol. 470, pp.63-83, 2002.

[6] Z. Borden and E. Meiburg, "Circulation-based models for Boussinesq internal bores," J. Fluid Mech., vol. 726, 2013.

[7] K. Ogden and K Helfrich, "Internal Hydraulic Jumps in two-layer flows with Upstream Shear," J. Fluid Mech. vol. 789, pp. 64-92, 2016.

[8] S. Popinet, "Gerris: a tree-based adaptive solver for the incompressible Euler equations in complex geometries," J. Comp. Phys. vol. 190, pp. 572-600, 2003.

[9] P. MacCready, W. R. Geyer, and H. Burchard, "Estuarine Exchange Flow is Related to Mixing through the Salinity Variance Budget," J. Phys. Oceanography, vol. 48, pp. 1375-1384, 2018.

[10] K. Ogden, "Internal Hydraulic Jumps with Upstream Shear," Ph.D. thesis, MIT WHOI, 2017.

[11] J. C. Wyngaard, "Turbulence in the Atmosphere," Cambridge University Press, 2010.

[12] H. Schlichting and K. Gersten, "Boundary-Layer Theory," Ninth Edition, Springer, 2016, p.173. 